\title{
Differential Transmission for Amplify-and-Forward Cooperative Communications
}

\author{
Thanongsak Himsoon, Student Member, IEEE, Weifeng Su, Member, IEEE, and K. J. Ray Liu, Fellow, IEEE
}

\begin{abstract}
In this letter, we propose a differential amplify-andforward (AF) transmission scheme for a two-user cooperative communications system. By efficiently combining signals from both direct and relay links, the proposed scheme provides superior performance compared to those of direct transmissions with either differential detection or coherent detection. While the exact biterror-rate (BER) formulation of the proposed scheme is not available currently, we provide, as a performance benchmark, an exact BER formulation and its simple bounds for a case of optimumcombining cooperation system with differential M-ary phase-shift keying (DMPSK) signals. The optimum power allocation is also determined based on the provided BER formulations. We show that the proposed differential cooperative transmission scheme together with the optimum power allocation yields comparable performance to the optimum-combining scheme. Simulation results show that the proposed differential scheme with optimum power allocation yields significant performance improvement over that with an equal power allocation scheme.
\end{abstract}

Index Terms-Amplify-and-forward protocol, cooperative diversity, differential modulation, performance analysis.

\section{INTRODUCTION}

D IFFERENTIAL detection together with the diversity combining technique is widely known as a mature technique that provides a good tradeoff between receiver complexity and performance. In differential phase-shift keying (DPSK) [1], efficient decoding relies on constant phase response of the channel from one time sample to the next. Due to the ability to bypass channel estimation at the receiver, the DPSK has been naturally extended to further exploit spatial diversity in the multiple-input multiple-output (MIMO) system [2], [3]. Recently, by taking advantage of the broadcasting nature of wireless networks, a novel cooperative communications scheme [4], [5] was introduced to explore the inherent spatial diversity through relay channels.

Recently, various cooperation protocols, e.g., amplify-andforward (AF) and decode-and-forward (DF) [4]-[7], have been proposed for wireless networks. Most of the works in [4]-[7] assume that the destination has perfect knowledge of channel state information (CSI) of all transmission links. While in some scenarios, e.g., the slow fading environment, the CSI is likely be acquired by the use of pilot symbols, it may not be possible in the fast fading environment. In addition, it is questionable how the destination can obtain source-relay channel perfectly through pilot signal forwarding without noise amplification. Moreover,

Manuscript received November 24, 2004; revised February 21, 2005. This work was supported in part under Grant CTA-ARL DAAD 190120011. The associate editor coordinating the review of this manuscript and approving it for publication was Dr. Mounir Ghogho.

T. Himsoon and K. J. R. Liu are with the Department of Electrical and Computer Engineering, University of Maryland, College Park, MD 20742 USA.

W. Su is with the Department of Electrical Engineering, State University of New York (SUNY) at Buffalo, Buffalo, NY 14260 USA.

Digital Object Identifier 10.1109/LSP.2005.853067 the computational overhead for channel estimation increases in proportional to the product of the number of transmit antennas and the number of relaying nodes.

To overcome such problems, a specific two-hop relay system using differential modulation has been investigated in [6]. Recently, a framework of noncoherent cooperative diversity has been proposed in [8] for the DF protocol employing frequency shift keying modulation. However, the framework does not fit the DPSK and the AF cooperation protocol.

In this letter, we propose a differential transmission scheme for the AF protocol in a two-user cooperative communications system. The scheme efficiently combines signals from all branches, in which only the long-term average of the received signals is required. As a performance benchmark, we provide an exact bit-error rate (BER) formulation and its simple bounds for the optimum-combining cooperation system with differntial M-ary PSK (DMPSK) signal. Based on the theoretical BER benchmark, we are able to obtain the optimum power allocation, which can be used to further improve the performance of the proposed scheme. Simulation results are shown to validate our proposed schemes and support our theoretical analysis.

\section{Proposed Differential Scheme}

Consider a two-user cooperative communications system employing the AF protocol. For differential transmission, the information is conveyed in the difference of the phases of two consecutive symbols. Specifically, information symbols to be broadcasted by the source is given by

$$
v_{m}=e^{j \phi_{m}}
$$

where $\left\{\phi_{m}\right\}_{m=0}^{M-1}$ is a set of $M$ information phases. In the case of DMPSK, $\phi_{m}$ can be specified as $\phi_{m}=2 \pi m / M$ for $m=$ $0,1, \ldots, M-1$. Instead of directly transmitting the information as in coherent transmission [4], [5], [7], the source node differentially encodes the information symbol $v_{m}$ as

$$
x^{\tau}=v_{m} x^{\tau-1}
$$

where $\tau$ is the time index, and $x^{\tau}$ is the differentially encoded symbol to be transmitted at time $\tau$. In the AF protocol, signal transmissions can be separated into two phases, and signals of all users are transmitted through orthogonal channels by using TDMA, FDMA, or CDMA schemes [4], [5].

In Phase 1, the source sends out the symbol $x^{\tau}$ with transmit power $P_{1}$. The corresponding received signals at the destination and the relay nodes can be expressed as

$$
y_{s, d}^{\tau}=\sqrt{P_{1}} h_{s, d}^{\tau} x^{\tau}+w_{s, d}^{\tau}
$$

and

$$
y_{s, r}^{\tau}=\sqrt{P_{1}} h_{s, r}^{\tau} x^{\tau}+w_{s, r}^{\tau}
$$

respectively. Here, $h_{s, d}^{\tau}$ and $h_{s, r}^{\tau}$ represent the channel coefficients from the source to the destination and that from the source to the relay, whereas $w_{s, d}^{\tau}$ and $w_{s, r}^{\tau}$ are additive noise. 
In Phase 2, the relay amplifies the received signal and forwards it to the destination with transmit power $P_{2}$. The received signal at the destination can be modeled as

$$
y_{r, d}^{\tau}=\sqrt{\tilde{P}_{2}} h_{r, d}^{\tau} y_{s, r}^{\tau}+w_{r, d}^{\tau}
$$

where $\tilde{P}_{2}$ represents normalized transmit power, $h_{r, d}^{\tau}$ is the channel coefficient from the relay to the destination, and $w_{r, d}^{\tau}$ is additive noise. In the Rayleigh fading environment, the channel coefficients $h_{s, d}^{\tau}, h_{s, r}^{\tau}$, and $h_{r, d}^{\tau}$ can be modeled as independent zero-mean complex Gaussian random variables with variances $\sigma_{s, d}^{2}, \sigma_{s, r}^{2}$ and $\sigma_{r, d}^{2}$, respectively. All of the fading coefficients are unknown to either the transmitter or the receiver, and they are assumed to be approximately the same over two symbol periods. The noise $w_{s, d}^{\tau}, w_{s, r}^{\tau}$ and $w_{r, d}^{\tau}$ are modeled as independent complex Gaussian random variables with zero means and variances $\mathcal{N}_{0}$. To ensure that the average transmit power of the relay node is $P_{2}$, the normalized power $\tilde{P}_{2}$ is specified as

$$
\tilde{P}_{2}=\frac{P_{2}}{P_{1} \sigma_{s, r}^{2}+\mathcal{N}_{0}}
$$

where the variance $\sigma_{s, r}^{2}$ can be obtained from long-term average of the received signals. The normalized power in (6) differs from its coherent counterpart in that the latter uses instantaneous fading amplitude, i.e., $\left|h_{s, r}\right|^{2}$, instead of $\sigma_{s, r}^{2}$.

At the destination, the received signal from the source through direct transmission in Phase $1\left(y_{s, d}^{\tau}\right)$ and that from the relay in Phase $2\left(y_{r, d}^{\tau}\right)$ are combined together, and then the combined output is differentially decoded. Based on the multichannel differentially coherent detection [9], the combined signal prior to differential decoding is

$$
y=a_{1}\left(y_{s, d}^{\tau-1}\right)^{*} y_{s, d}^{\tau}+a_{2}\left(y_{r, d}^{\tau-1}\right)^{*} y_{r, d}^{\tau}
$$

where the coefficients $a_{1}$ and $a_{2}$ are given by

$$
\begin{aligned}
& a_{1}=\frac{1}{\mathcal{N}_{0}} \\
& a_{2}=\frac{P_{1} \sigma_{s, r}^{2}+\mathcal{N}_{0}}{\mathcal{N}_{0}\left(P_{1} \sigma_{s, r}^{2}+P_{2} \sigma_{r, d}^{2}+\mathcal{N}_{0}\right)}
\end{aligned}
$$

respectively. Without acquiring CSI, the decoder uses the sufficient statistics given in (7) to decode information as

$$
\hat{m}=\arg \max _{m=0,1, \ldots, M-1} \operatorname{Re}\left\{v_{m}^{*} y\right\} .
$$

\section{ANALYSIS AND DISCUSSIONS}

While the exact BER formulation that is applicable for the multichannel differential scheme with arbitrary-weight combining as in (8) is currently not available, we provide a BER formulation for an ideal maximum ratio combining that is obtained by using the optimum weights [10]

$$
\begin{aligned}
& \hat{a}_{1}=\frac{1}{\mathcal{N}_{0}} \\
& \hat{a}_{2}=\frac{P_{1} \sigma_{s, r}^{2}+\mathcal{N}_{0}}{\mathcal{N}_{0}\left(P_{1} \sigma_{s, r}^{2}+P_{2}\left|h_{r, d}\right|^{2}+\mathcal{N}_{0}\right)} .
\end{aligned}
$$

Even though the optimum weights are not practical for the proposed scheme since they rely on the CSI $\left|h_{r d}\right|^{2}$, the performance evaluation based on such optimum weights can be used as a performance benchmark. We will show in the next section that the performance of the proposed scheme with optimum power allocation is close to the ideal combining benchmark.
With the optimum weights $\hat{a}_{1}$ and $\hat{a}_{2}$, the instantaneous signal-to-noise ratio (SNR) of the combiner output is $\gamma=\gamma_{1}+\gamma_{2}$ [10], where

$$
\gamma_{1}=\frac{P_{1}\left|h_{s, d}\right|^{2}}{\mathcal{N}_{0}}
$$

and

$$
\gamma_{2}=\frac{P_{1} P_{2}\left|h_{s, r}\right|^{2}\left|h_{r, d}\right|^{2}}{\mathcal{N}_{0}\left(P_{1} \sigma_{s, r}^{2}+P_{2}\left|h_{r, d}\right|^{2}+\mathcal{N}_{0}\right)}
$$

in which the time index $\tau$ is omitted in this section to simplify the notations. By using the BER expression for two-channel DMPSK in [1], the conditional BER is given by

$$
P_{b \mid \gamma}=\frac{1}{16 \pi} \int_{-\pi}^{\pi} f(\theta) \exp [-\alpha(\theta) \gamma] d \theta
$$

where

$$
f(\theta)=\frac{b^{2}\left(1-\beta^{2}\right)[3+\cos (2 \theta)-(\beta+1 / \beta) \sin \theta]}{2 \alpha(\theta)}
$$

and

$$
\alpha(\theta)=\frac{b^{2}\left(1+2 \beta \sin \theta+\beta^{2}\right)}{2} .
$$

Here, $\beta=a / b$ is a constant in which $a$ and $b$ depend on modulation size, specifically, $a=10^{-3}$ and $b=\sqrt{2}$ for DBPSK modulation and $a=\sqrt{2-\sqrt{2}}$ and $b=\sqrt{2+\sqrt{2}}$ for DQPSK modulation [1]. The values of $a$ and $b$ for larger modulation sizes can be obtained by using the result in [9]. Averaging the conditional BER over the Rayleigh fading channels $h_{s, d}, h_{s, r}$ and $h_{r, d}$ results in the average BER

$$
P_{b}=\frac{1}{16 \pi} \int_{-\pi}^{\pi} f(\theta) \mathcal{M}_{\gamma_{1}}(\theta) \mathcal{M}_{\gamma_{2}}(\theta) d \theta
$$

where $\mathcal{M}_{\gamma_{i}}(\theta)=\int_{-\infty}^{+\infty} e^{-\alpha(\theta) \lambda} p_{\gamma_{i}}(\lambda) d \lambda$ denotes the MGF of the SNR $\gamma_{i}$ for $i=1,2$. The BER formulation in (14) can be further calculated as follows.

For Rayleigh independent fading channels, $\left|h_{s, d}\right|^{2},\left|h_{s, r}\right|^{2}$ and $\left|h_{r, d}\right|^{2}$ areindependentexponential random variables with parameter $1 / \sigma_{s, d}^{2}, 1 / \sigma_{s, r}^{2}$ and $1 / \sigma_{r, d}^{2}$, respectively. Thus, we have

$$
\mathcal{M}_{\gamma_{1}}(\theta)=\frac{1}{1+k_{s, d}(\theta)}
$$

where $k_{s, d}(\theta) \triangleq \alpha(\theta) P_{1} \sigma_{s, d}^{2} / \mathcal{N}_{0}$. Observe that $\mathcal{M}_{\gamma_{2}}(\theta)$ depends on both $\left|h_{s, r}\right|^{2}$ and $\left|h_{r, d}\right|^{2}$. By averaging over $\left|h_{s, r}\right|^{2}$, after some manipulations, we can express $\mathcal{M}_{\gamma_{2}}(\theta)$ as

$$
\begin{aligned}
& \mathcal{M}_{\gamma_{2}}(\theta)=\frac{1}{1+k_{s, r}(\theta)}\left(1+\frac{k_{s, r}(\theta)}{1+k_{s, r}(\theta)} \frac{P_{1} \sigma_{s, r}^{2}+\mathcal{N}_{o}}{P_{2}}\right. \\
& \text { where } \\
& \left.\qquad \frac{1}{\sigma_{r, d}^{2}} \int_{0}^{\infty} \frac{\exp \left(-u / \sigma_{r, d}^{2}\right)}{u+R(\theta)} d u\right) \\
& R(\theta) \triangleq \frac{P_{1} \sigma_{s, r}^{2}+\mathcal{N}_{o}}{P_{2}\left(1+k_{s, r}(\theta)\right)}
\end{aligned}
$$

in which $k_{s, r}(\theta)$ is given by $k_{s, r}(\theta) \triangleq \alpha(\theta) P_{1} \sigma_{s, r}^{2} / \mathcal{N}_{0}$. Finally, by substituting (15) and (16) into (14), we obtain a BER expression that involves only double integration. In what follows, we can further obtain single-integral BER lower bound, single-integral BER upper bound, and their corresponding simple BER approximations that involve no integration.

The BER expression in (14) can be upper bounded by bounding $\mathcal{M}_{\gamma_{2}}(\theta)$ in (16). We can see from (17) that $R(\theta)$ 
reaches its minimum value when $\alpha(\theta)$ attains its maximum at $\theta=\pi / 2$, i.e., $\alpha(\theta) \leq\left(b^{2}(1+\beta)^{2}\right) / 2$. Accordingly, the minimum value of $R(\theta)$ is given by

$$
\begin{aligned}
R(\theta) \geq \frac{P_{1} \sigma_{s, r}^{2}+\mathcal{N}_{o}}{P_{2}} & \\
\times & {\left[1+\frac{P_{1} \sigma_{s, r}^{2} b^{2}(1+\beta)^{2}}{2 \mathcal{N}_{0}}\right]^{-1} \triangleq R_{\text {min }} . }
\end{aligned}
$$

Substituting $R(\theta)$ in (16) with $R_{\min }$, the BER upper bound is given by

$$
\begin{aligned}
P_{b} \leq \frac{1}{16 \pi} \int_{-\pi}^{\pi} f(\theta) \frac{1}{\left[1+k_{s, d}(\theta)\right]\left[1+k_{s, r}(\theta)\right]} \\
\times\left(1+\frac{P_{1} \sigma_{s, r}^{2}+1}{P_{2} \sigma_{r, d}^{2}} \frac{k_{s, r}(\theta)}{1+k_{s, r}(\theta)} Z_{\max }\right) d \theta
\end{aligned}
$$

in which $Z_{\max }=\int_{0}^{\infty} \exp \left(-u / \sigma_{r, d}^{2}\right)\left[u+R_{\min }\right]^{-1} d u$ is a constant that can be easily obtained for any given values of $\sigma_{r, d}^{2}$ and $R_{\min }$. The upper bound $P_{\mathrm{ub}}$ in (19) involves only single integration, and it is simpler than the exact BER provided in (14).

To get more insights, we further simplify the BER upper bound in (19) as follows. For high enough SNR, we can ignore all 1's in the denominator of (19). This results in

$$
\begin{aligned}
& P_{b} \leq \frac{\left(P_{1} \sigma_{s, r}^{2}+1\right) Z_{\max }+P_{2} \sigma_{r, d}^{2}}{P_{1}^{2} P_{2} \sigma_{s, d}^{2} \sigma_{s, r}^{2} \sigma_{r, d}^{2}} \mathcal{N}_{0}^{2} C(\beta, \theta) \\
& \text { where } \\
& C(\beta, \theta)=\frac{1}{8 \pi b^{4}} \\
& \times \int_{-\pi}^{\pi} \frac{\left(1-\beta^{2}\right)[3+\cos (2 \theta)-(\beta+1 / \beta) \sin \theta]}{\left(1+2 \beta \sin \theta+\beta^{2}\right)^{3}} d \theta
\end{aligned}
$$

is a constant that depends on modulation size.

The BER lower bound can be obtained in a similar way as the case of the BER upper bound. In this case, $R(\theta)$ reaches its maximum value when $\alpha(\theta)$ attains its minimum at $\theta=-\pi / 2$, i.e., $\alpha(\theta) \geq\left(b^{2}(1-\beta)^{2}\right) / 2$. Accordingly, the maximum value of $R(\theta)$ is given by

$$
\begin{aligned}
R(\theta) \leq \frac{P_{1} \sigma_{s, r}^{2}+\mathcal{N}_{o}}{P_{2}} & \\
\times & {\left[1+\frac{P_{1} \sigma_{s, r}^{2} b^{2}(1-\beta)^{2}}{2 \mathcal{N}_{0}}\right]^{-1} \triangleq R_{\max } . }
\end{aligned}
$$

Therefore, the lower bound is given by

$$
\begin{aligned}
P_{b} \geq \frac{1}{16 \pi} \int_{-\pi}^{\pi} f(\theta) \frac{1}{\left[1+k_{s, d}(\theta)\right]\left[1+k_{s, r}(\theta)\right]} \\
\quad \times\left(1+\frac{P_{1} \sigma_{s, r}^{2}+1}{P_{2} \sigma_{r, d}^{2}} \frac{k_{s, r}(\theta)}{1+k_{s, r}(\theta)} Z_{\min }\right) d \theta
\end{aligned}
$$

in which $Z_{\min }=\int_{0}^{\infty} \exp \left(-u / \sigma_{r, d}^{2}\right)\left[u+R_{\max }\right]^{-1} d u$ is a constant that can be easily obtained for any given values of $\sigma_{r, d}^{2}$ and $R_{\max }$.

By ignoring all 1's in the denominator of (22), the resulting BER approximation of (22) can be expressed as

$$
P_{b} \approx \frac{\left(P_{1} \sigma_{s, r}^{2}+1\right) Z_{\min }+P_{2} \sigma_{r, d}^{2}}{P_{1}^{2} P_{2} \sigma_{s, d}^{2} \sigma_{s, r}^{2} \sigma_{r, d}^{2}} \mathcal{N}_{0}^{2} C(\beta, \theta)
$$

The approximated BER in (23) is tight at high SNR. We will show in the simulation results that the lower bound and its simple approximation are tight in a high SNR region.

Based on the analysis in this section, we will show in the next section that the BER expression provides a performance bench-

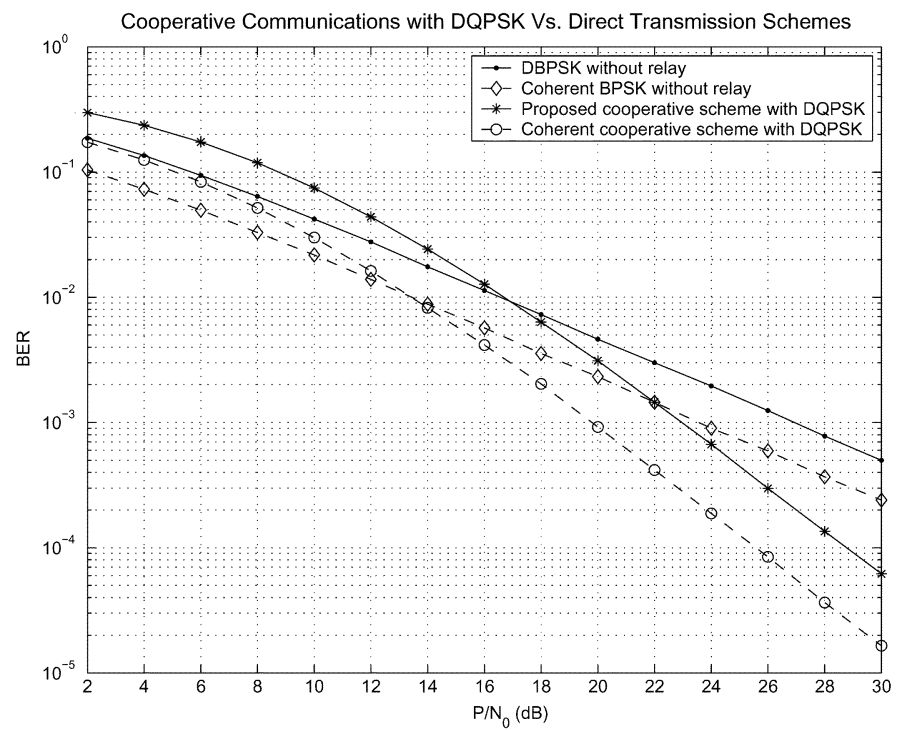

Fig. 1. Cooperative communication system with DQPSK signals $\sigma_{s, d}^{2}=$ $\sigma_{s, r}^{2}=\sigma_{r, d}^{2}=1, P_{1} / P=0.7$, and $P_{2} / P=0.3$.

mark for our proposed scheme. In addition, the BER upper bound and lower bound together with their simple approximations are close to the performance benchmark. Moreover, the optimum power allocation based on the BER approximations can be used to further improve the performance of the proposed scheme.

\section{Simulation Results}

We perform computer simulations for the two-user cooperation systems employing the AF protocol with DQPSK signals. The channel fading coefficients are independent between communication links but time correlated according to the Jakes' model [11], in which the Doppler frequency is $f_{D}=75 \mathrm{~Hz}$ and normalized fading parameter is $f_{D} T_{s}=0.0025$, where $T_{s}$ is the sampling period. The noise variance is unity $\left(\mathcal{N}_{0}=1\right)$. We plot the performance curves in terms of average BER versus $P / \mathcal{N}_{0}$, where $P$ is the total transmit power. We assume that the power allocation at the source and relay nodes is fixed as $P_{1}+P_{2}=P$.

Fig. 1 compares simulated performances of the proposed differential cooperative scheme to differential direct transmission and its coherent counterpart. The channel variances are chosen as $\sigma_{s d}^{2}=\sigma_{s, r}^{2}=\sigma_{r, d}^{2}=1$, and power ratios are given by $P_{1}=0.7 P$ and $P_{2}=0.3 P$. For fair comparison in terms of bandwidth efficiency, we simulate the direct transmission schemes with DBPSK signals. It is apparent that the proposed differential cooperative scheme achieves higher diversity orders than the DBPSK with direct transmission at high SNRs. We observe about a $4 \mathrm{~dB}$ performance gain at a BER of $10^{-3}$. In addition, at SNRs higher than $22 \mathrm{~dB}$, the proposed differential scheme outperforms the directly transmitted BPSK with coherent detection.

We illustrate in Fig. 2 the theoretical BER benchmark in comparison to the simulated performance of our proposed scheme in the case of $\sigma_{s, d}^{2}=\sigma_{s, r}^{2}=\sigma_{r, d}^{2}=1$ and $P_{1}=0.7 P$ and $P_{2}=0.3 P$. We can see that the BER expression with optimum weights in (10) is close to the simulated performance of the proposed scheme. The upper bound (19) and its simple approximation (20) are asymptotically parallel to the exact BER benchmark. The lower bound in (22) is tight to the simulated performance and the exact BER benchmark. The simple approximate BER in (23) is loose at low SNR but asymptotically tight in the reasonably high SNR range. 


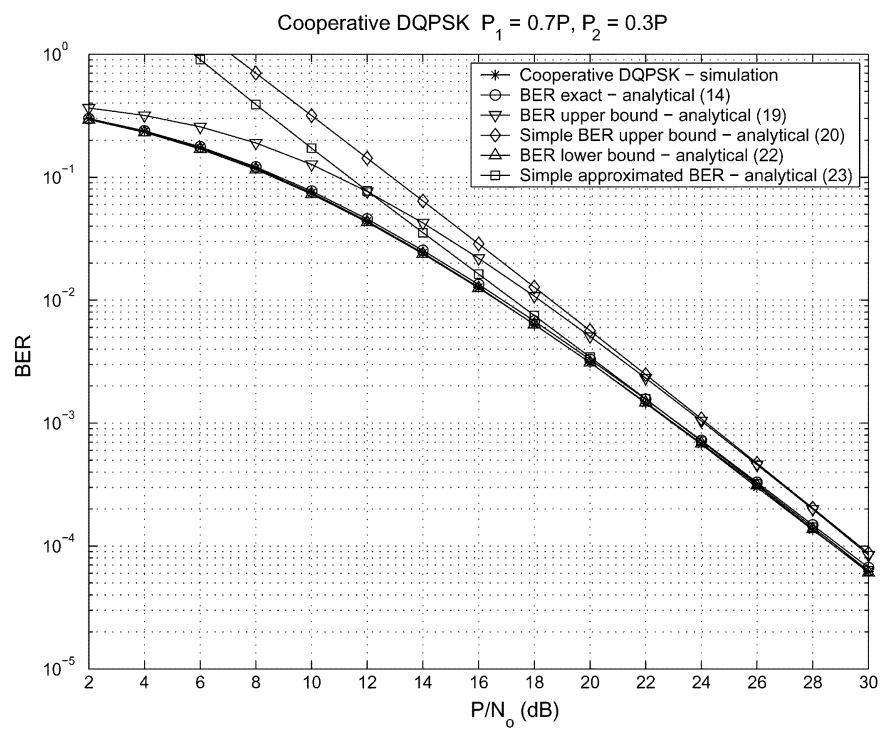

Fig. 2. Theoretical BER benchmark and the simulated performance of the proposed scheme $\sigma_{s, d}^{2}=\sigma_{s, r}^{2}=\sigma_{r, d}^{2}=1$.

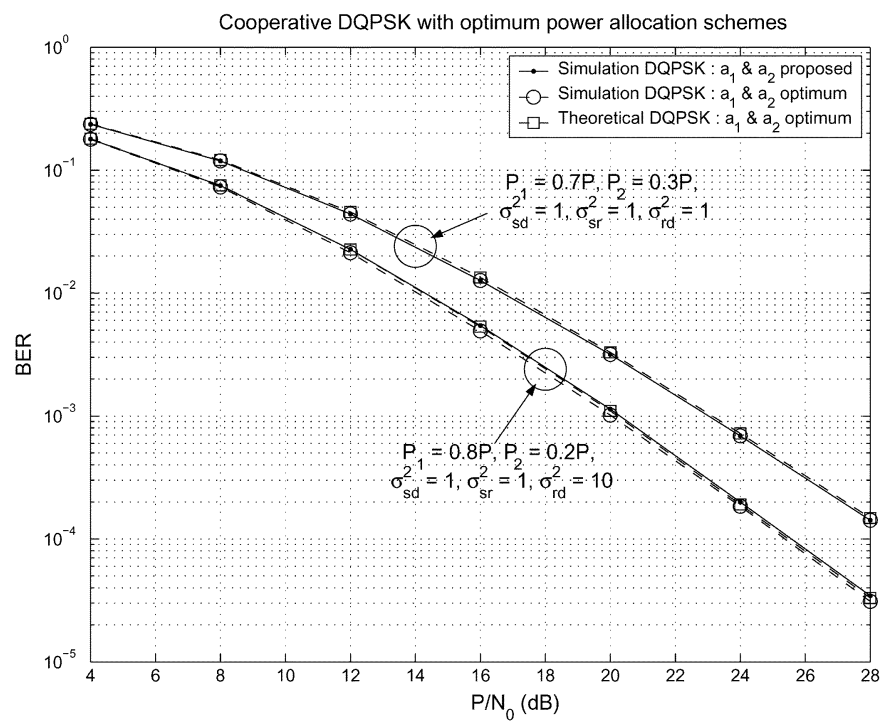

Fig. 3. Performance when optimum power allocation is applied.

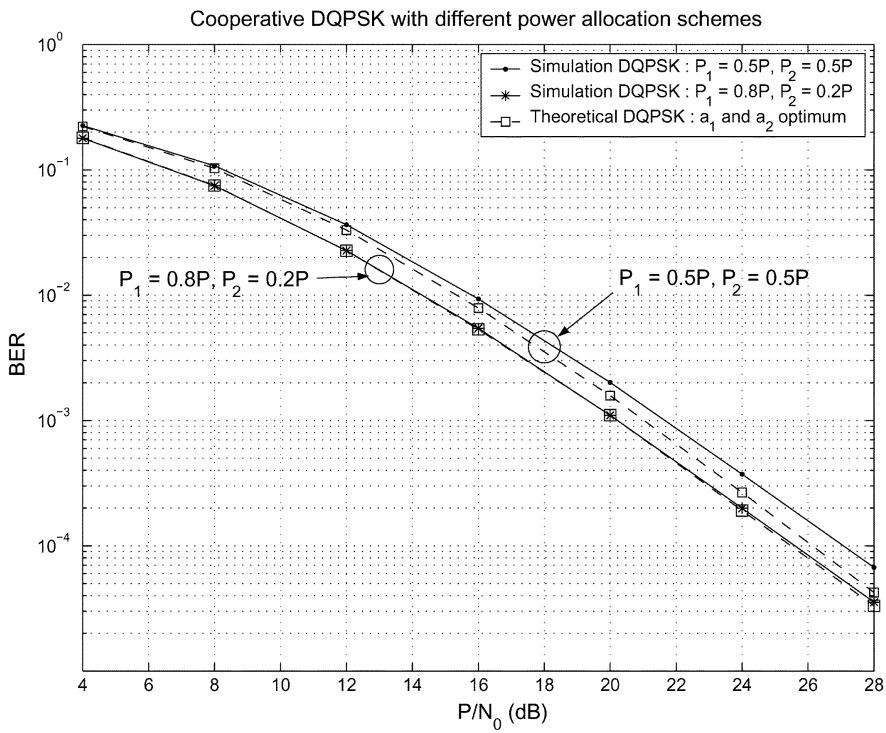

Fig. 4. Performance of the proposed scheme with optimum and equal power allocation $\sigma_{s, d}^{2}=\sigma_{s, r}^{2}=1$ and $\sigma_{r, d}^{2}=10$.
We numerically find the optimum power allocation based on the BER benchmark at an SNR of $20 \mathrm{~dB}$. Fig. 3 depicts performances of the proposed scheme and the benchmark scheme when the power is optimally allocated. We consider the scenarios when all channel variances are unity and when $\sigma_{s, d}^{2}=\sigma_{s, r}^{2}=1$ and $\sigma_{r, d}^{2}=10$. We can see that regardless of the channel conditions, both schemes yield comparable performances, which are close to the theoretical BER benchmark. This interesting result provides a key concept in allocating power among users. Specifically, in order to balance the link qualities, lower power should be allocated to the link with larger channel variance, while higher power should be put in the link with smaller channel variance. As can be seen in Fig. 4, the power loading $\hat{P}_{1}$ and $\hat{P}_{2}$ yields the improvement of about 1.4 $\mathrm{dB}$ at a BER of $10^{-3}$ over the one with equal power allocation.

\section{CONCLUSION}

We proposed a differential AF scheme for a two-user cooperative communications system. The proposed scheme with DQPSK signals provided a $4 \mathrm{~dB}$ performance improvement at a BER of $10^{-3}$ over that of the DBPSK direct transmission scheme. In comparison to the coherent detection without relay, the proposed scheme provided a practical alternative with lowercomplexity and simpler implementation. In addition, simulation results showed that the performance of the proposed scheme is superior to that of direct transmission with coherent detection at SNRs higher than $22 \mathrm{~dB}$. This is due to the fact that the cooperative communications provide more diversity gain than the direct transmission schemes. While the BER analysis of the proposed scheme is not tractable, we provided the exact BER expression based on optimum combining weights, and it is considered as a performance benchmark for our proposed scheme. By using the optimum power allocation based on the provided BER expression, the proposed scheme is able to achieve comparable performance to the benchmark scheme in any channel variances of all links. Moreover, the performance of optimum power strategy outperforms that of equal power scheme of about $1.4 \mathrm{~dB}$ at a BER of $10^{-3}$.

\section{REFERENCES}

[1] M. K. Simon and M.-S. Alouini, "A unified approach to the probability of error for noncoherent and differentially coherent modulations over generalized fading channels," IEEE Trans. Commun., vol. 46, no. 12, pp. $1625-1638$, Dec. 1998.

[2] B. M. Hochwald and W. Sweldens, "Differential unitary space-time modulation," IEEE Trans. Commun., vol. 48, no. 12, pp. 2041-2052, Dec. 2000.

[3] B. L. Hughes, "Differential space-time modulation," IEEE Trans. Inf. Theory, vol. 46, no. 11 , pp. 2567-2578, Nov. 2000 .'

[4] J. N. Laneman, D. N. C. Tse, and G. W. Wornell, "Cooperative diversity in wireless networks: Efficient protocols and outage behavior," IEEE Trans. Inf. Theory, vol. 50, no. 12, pp. 3062-3080, Dec. 2004.

[5] J. N. Laneman and G. W. Wornell, "Distributed space-time coded protocols for exploiting cooperative diversity in wireless networks," IEEE Trans. Inf. Theory, vol. 49, no. 10, pp. 2415-2525, Oct. 2003.

[6] M. O. Hasna and M.-S. Alouini, "Performance analysis of two-hop relayed transmissions over Rayleigh fading channels," in Proc. IEEE Veh. Tech. Conf. , vol. 4, Nov. 2003, pp. 1992-1996.

[7] W. Su, A. K. Sadek, and K. J. R. Liu, "SER performance analysis and optimum power allocation for decode-and-forward cooperation protocol in wireless networks," in Proc. IEEE WCNC, New Orleans, LA, Mar. 2005, pp. 984-989.

[8] D. Chen and J. N. Laneman, "Noncoherent demodulation for cooperative diversity in wireless systems," in IEEE Global Comm. Conf., Dallas, TX, Nov. 2004, pp. 31-35.

[9] J. G. Proakis, Digital Communications, 4th ed. New York: McGrawHill, 2000.

[10] D. G. Brennan, "Linear diversity combining techniques," Proc. IEEE, vol. 91, no. 2, pp. 331-356, Feb. 2003.

[11] W. C. Jakes, Microwave Mobile Communications. Piscataway, NJ: IEEE Press, 1993. 